\title{
Association between PEG3 DNA methylation and high-grade cervical intraepithelial neoplasia
}

Claire Bosire $^{1 *}$ D, Adriana C. Vidal ${ }^{2}$, Jennifer S. Smith ${ }^{3}$, Dereje Jima ${ }^{4}$, Zhiqing Huang ${ }^{5}$, David Skaar ${ }^{4}$, Fidel Valea ${ }^{6}$, Rex Bentley ${ }^{7}$, Margaret Gradison ${ }^{8}$, Kimberly S. H. Yarnall ${ }^{8}$, Anne Ford ${ }^{5}$, Francine Overcash ${ }^{8}$, Susan K. Murphy ${ }^{5}$ and Cathrine Hoyo ${ }^{4}$

\begin{abstract}
Background: Epigenetic mechanisms are hypothesized to contribute substantially to the progression of cervical intraepithelial neoplasia (CIN) to cervical cancer, although empirical data are limited.

Methods: Women $(n=419)$ were enrolled at colposcopic evaluation at Duke Medical Center in Durham, North Carolina. Human papillomavirus (HPV) was genotyped by HPV linear array and CIN grade was ascertained by biopsy pathologic review. DNA methylation was measured at differentially methylated regions (DMRs) regulating genomic imprinting of the IGF2/H19, IGF2AS, MESTIT1/MEST, MEG3, PLAGL1/HYMAI, KVDMR and PEG10, PEG3 imprinted domains, using Sequenom-EpiTYPER assays. Logistic regression models were used to evaluate the associations between HPV infection, DMR methylation and CIN risk overall and by race.

Results: Of the 419 participants, 20 had CIN3+, 52 had CIN2, and 347 had $\leq$ CIN1 (CIN1 and negative histology). The median participant age was 28.6 (IQR:11.6) and 40\% were African American. Overall, we found no statistically significant association between altered methylation in selected DMRs and CIN2+ compared to $\leq$ CIN1. Similarly, there was no significant association between DMR methylation and CIN3+ compared to $\leq$ CIN2. Restricting the outcome to CIN2+ cases that were HR-HPV positive and p16 staining positive, we found a significant association with PEG3 DMR methylation (OR: 1.56 95\% Cl: 1.03-2.36).
\end{abstract}

Conclusions: While the small number of high-grade CIN cases limit inferences, our findings suggest an association between altered DNA methylation at regulatory regions of PEG3 and high grade CIN in high-risk HPV positive cases.

Keywords: Cervical intraepithelial neoplasia, Gene methylation, Imprinted gene, Human papillomavirus

\section{Background}

Screening and treatment of cervical intraepithelial neoplasia (CIN) has significantly reduced invasive cervical cancer (ICC) incidence and mortality [1]. However, an estimated 13,800 new ICC cases and 4290 deaths were expected in the United States in 2020 [2]. ICC continues

\footnotetext{
* Correspondence: cbosire@email.unc.edu

'Department of Health Behavior, Gillings School of Global Public Health, University of North Carolina, Chapel Hill, NC, USA

Full list of author information is available at the end of the article
}

to disproportionally affect Hispanic and Black women as compared to White and Asian-American women [3].

Current cervical cancer screening guidelines include cytology-based screening, with the addition of high-risk human papillomavirus (HPV) testing in women 30 years and older to increase sensitivity for the detection of high grade CIN (CIN2+) or cancer [4]. Cytology-based tests have relatively low single-use sensitivity for detecting CIN2+ which could delay appropriate treatment $[5,6]$. HPV testing is more sensitive than cytology in detecting

(c) The Author(s). 2021 Open Access This article is licensed under a Creative Commons Attribution 4.0 International License, which permits use, sharing, adaptation, distribution and reproduction in any medium or format, as long as you give appropriate credit to the original author(s) and the source, provide a link to the Creative Commons licence, and indicate if changes were made. The images or other third party material in this article are included in the article's Creative Commons licence, unless indicated otherwise in a credit line to the material. If material is not included in the article's Creative Commons licence and your intended use is not permitted by statutory regulation or exceeds the permitted use, you will need to obtain permission directly from the copyright holder. To view a copy of this licence, visit http://creativecommons.org/licenses/by/4.0/ The Creative Commons Public Domain Dedication waiver (http://creativecommons.org/publicdomain/zero/1.0/) applies to the data made available in this article, unless otherwise stated in a credit line to the data. 
CIN2+ but has relatively lower specificity for CIN2+ [7], detecting transient infections which could lead to unnecessary referrals to colposcopy. Besides a strategy to triage HPV positive women, molecular markers that can improve the prediction of progression of CIN to ICC are needed. Further, to increase the participation of underscreened populations, screening strategies that include vaginal self-sampling with high-risk HPV (HR-HPV) testing are a viable alternative [8]. It is important to identify molecular markers to triage HPV positive women using self-sampling devices in the absence of cervical cytology.

Methylation biomarkers are promising tools for the detection of CIN2+ $[9,10]$. DNA methylation plays an important role in the regulation of gene expression and cancer development [11]. In high-grade precancer and cancer, imprinted differentially methylated regions (DMRs) are often deregulated, showing gains or losses in methylation that affect changes in expression and sometimes alter imprint status [12]. DNA methylation changes can result in either silencing of the only active allele or, aberrantly activating expression of the normally silenced allele, which may double the gene dosage [13].

DNA methylation in genes and HPV testing have been investigated as triage methods to improve risk stratification of HR-HPV positive women. Studies summarizing the performance of DNA methylation in CIN2 and CIN3 detection have reported significantly higher DNA methylation in $\mathrm{CIN} 2+$ and CIN3+ compared to $\leq \mathrm{CIN} 1$ $[10,14]$. We previously reported that aberrant methylation at DMRs that regulate the expression of imprinted genes including Insulin-like Growth Factor 2 (which expresses IGF2, a potent mitogen), Paternally Expressed Gene 1/Mesoderm-Specific Transcript (PEG1/MEST) and Paternally Expressed Gene 3 (PEG3) were independent predictors of CIN $2 / 3$ and ICC in a cross-sectional study of Tanzanian women with CIN and ICC $[15,16]$. We also reported a negative association between CIN1 regression and methylation at Insulin-Like Growth Factor 2, Antisense (IGF2AS) and Paternally Expressed Gene 10 (PEG10) DMR in a study of CIN1 cases drawn from the Duke University Cervical Intraepithelial Neoplasia Cohort Study (CINCS) [17]. Preliminary analyses have found dysregulated expression of imprinted genes involved in tumor suppression (e.g., Pleiomorphic Adenoma Gene-Like 1 (PLAGL1) and Hydatidiform Mole Associated and Imprinted (HYMAI)) in cervical cancer specimen compared to normal cervical tissue [18], and Maternally Expressed Gene 3 (MEG3) hypermethylation has been implicated as a potential biomarker in cervical cancer [19]. While studies in voltage-gated potassium channels $(K v) D M R$ are limited, changes in methylation at $K v$ DMR have been positively associated with breast [20] and colorectal cancer [21].
Here, we examine whether aberrant DNA methylation at DMRs regulating genomic imprinting of $I G F 2 / H 19$, IGF2AS, MESTIT1/MEST, MEG3, PLAGL1/HYMAI, Kv $D M R$ and PEG10, PEG3, are associated with CIN $(\mathrm{CIN} 2+$ vs $\leq \mathrm{CIN} 1$ and $\mathrm{CIN} 3+$ vs $\leq \mathrm{CIN} 2)$ in women participating in the multiethnic CINCS [22] overall, and in race-stratified analyses.

\section{Methods}

\section{Study participants}

Study participants were recruited from all 10 Duke University and Duke colposcopy clinics in Durham County, North Carolina, from 2010 to 2012, as previously described [22]. Briefly, all clinics used the same study protocol and the Duke University Pathology Laboratory for cytologic and histologic evaluation. To be eligible, study participants were initially screened for cervical abnormalities with the Thin-Prep liquid-based cytology test $\left(\mathrm{Cytyc}^{\circ}\right)$. Inclusion criteria were: a visit to one of 10 colposcopy clinics following an abnormal Pap test of at least low grade squamous intraepithelial lesion (LSIL), age 18 years or older, and English or Spanish speaking. Questionnaires were written in English and a Spanishspeaking coordinator assisted and interpreted the questionnaire content to Spanish-speaking study participants. The self- and interviewer-administered instruments were identical in content. Women who did not intend to receive follow-up care in one of the 10 colposcopy clinics or moved out of the area for other reasons were excluded. Of the 1657 women with cytological abnormalities approached in the colposcopy clinic, 1303 were enrolled, a response rate of $79 \%$. This study was approved by Duke University School of Medicine Institutional Review Board and informed consent was obtained from each study participant prior to enrollment in the study.

\section{Data collection}

A standardized questionnaire that was either self- or interviewer-administered solicited information on risk factors such as: age, race, parity, yearly income, cigarette smoking, contraceptive use, dietary and sexual habits. Age was treated as a continuous variable; race/ethnicity was categorized as non-Hispanic Black; non-Hispanic White, Hispanic and other; parity was nulliparous or parous; HPV-DNA status was none, high-risk or lowrisk HPV; and current smoking, contraceptive use and previous HPV vaccination were yes or no categories.

\section{Specimens}

At the enrollment visit, exfoliation and collection of cervical cells was performed using a spatula and cytobrush. Cervical exfoliated cells were suspended in a vial containing ThinPrep solution (Hologic ${ }^{\circ}$, Malborough, MA, 
USA) for cytological assessment. Colposcopy-directed biopsies were also obtained from the lesions. All specimens were tested for adequacy using the 2012 ASCCP guidelines [23]. The specimens were stored at $4{ }^{\circ} \mathrm{C}$ prior to HPV testing.

\section{Ascertainment of CIN and HPV genotyping}

The biopsies underwent pathologic review to ascertain the presence of CIN. The hematoxylin and eosin-stained slides of individuals with cytological abnormalities were read by the study pathologist's (RB) laboratory.

Testing for 37 HPV DNA subtypes was done at Johns Hopkins University, as previously described [22]. Following DNA extraction, HPV status was determined by targeted amplification of a $450 \mathrm{bp}$ region of the HPV L1 genome using PGMY09/PGMY11 primers. Amplification of the human $\beta$-globin gene was included as an internal control for sample sufficiency. HPV genotyping was performed using HPV Linear Array (Roche Diagnostics, Branchburg, NJ, USA) [24, 25]; HPV genotypes 16, $18,31,33,35,39,45,51,52,56,58,59,66$, and 68 were considered high-risk or oncogenic genotypes, whereas HPV 6, 11, 26, 40, 42, 53, 54, 55, 61, 62, 64, 67, 69, 70, $72,73,81,82,83$ and 84 were considered low-risk (LR) HPV types [26, 27].

\section{DMR DNA methylation}

DNA methylation was measured at differentially methylated regions (DMRs) regulating genomic imprinting of IGF2/H19, IGF2AS, MESTIT1/MEST, Kv DMR, MEG3, PLAGL1/HYMAI and PEG3 and PEG10 imprinted domains using Sequenom (San Diego, CA) EpiTYPER assays with the primers shown in Additional file 1. For each sample, $800 \mathrm{ng}$ of DNA isolated from the exfoliated cervical cells were bisulfite converted using the EZ-96 DNA methylation kit (Zymo Research Corporation, Irvine, CA) to convert unmethylated DNA cytosine bases to uracil bases, leaving methylated cytosines unchanged as per manufacturer's protocol. The bisulfite converted DNA was eluted into 40ul of elution buffer and transferred into 384-well plates. PCR was carried out using $20 \mathrm{ng}$ bisulfite-converted DNA in a 10ul reaction volume with HotStarTaq DNA Polymerase (Qiagen; Valencia, $\mathrm{CA})$. The PCR products were then treated with shrimp alkaline phosphatase (SAP, Sequenom, San Diego, CA) followed by transcription and $\mathrm{T}$ cleavage reactions according to the protocol from Sequenom. The cleanup and sequencing were performed according to the EpiTYPER user guide (Sequenom). The average methylation percentage from the $\mathrm{CpG}$ sites included in each DMR was used for analysis.

\section{Statistical analyses}

Analyses were limited to 419 participants who had DNA methylation data, confirmed CIN status and covariate data including race/ethnicity and age. The primary contrast was CIN2+ vs $\leq \mathrm{CIN} 1$. We also evaluated CIN3+ vs $\leq \mathrm{CIN} 2$. Chi-squared and Fisher's exact tests were used to compare socio-demographic characteristics of women with $\leq \mathrm{CIN} 1$ (CIN1 or no evidence of CIN) to those with CIN2, and CIN3+. HPV infections were grouped according to potential oncogenicity [26, 27] using the Bethesda criteria [28], as previously described [22]. Samples that failed assays owing to suboptimal DNA quality were excluded from analysis. A total of eight imprinted DMRs were considered a priori with five CpG sites for the IGF2/H19 DMR; 10 CpGs for the IGF2AS DMR; 31 CpGs for the MESTIT1/MEST DMR; 27 CpG sites for the Kv DMR; $31 \mathrm{CpG}$ sites for the MEG3 DMR; eight CpG sites for the PLAGL1/HYMAI DMR; 11 CpG sites for the PEG10 DMR; and $12 \mathrm{CpG}$ sites for the PEG3 DMR. We calculated summary methylation percentages across each candidate region. Mann-Whitney test was used to compare methylation differences across groups and receiver operating characteristic (ROC) curve analyses were used to evaluate how well methylation identified women with $\mathrm{CIN} 2+$ or CIN3 + .

We used logistic regression models to estimate odds ratios (OR) and corresponding 95\% confidence intervals (CI) for the association between CIN (CIN2+ vs $\leq$ CIN1) and changes in DMR methylation. We also evaluated the association between methylation changes and CIN3+ vs $\leq$ CIN2. All models included methylation in $10 \%$ increments with adjustments made for race/ethnicity, age, parity, current smoking and HPV infection. In stratified analysis, we computed the OR and 95\% CI for CIN2+ and CIN3+ and changes in DMR methylation separately for white and black women, adjusting for age, parity, current smoking and HPV infection. In additional analysis, we evaluated the association in $\mathrm{CIN} 2+$ cases that were 16 positive and HR-HPV positive.

We also estimated regression coefficients for the association between DMR methylation and HPV infection using mixed methods to allow for unconstrained model entry of individual CpGs at each DMR, with no HPV infection serving as the referent category. Statistical analyses were conducted using SAS 9.4 (SAS Institute, Cary, NC).

\section{Results}

\section{Study participants}

These results are based on the 419 participants who had DNA methylation data, confirmed CIN status and covariate data. There were seven beta-globin negative samples which were excluded from the analysis. Fourteen HR- 
HPV types were detected in 309 (74\%) of participants, as single or multiple infections. Overall, the five most commonly detected HR-HPV types were HPV16 (55/419, $13 \%)$ and HPV66 $(n=55,13 \%), \operatorname{HPV} 52(n=48,11.5 \%)$, HPV51 $(n=46,11 \%)$, and HPV39 $(n=44,10.5 \%)$. The distribution of other HR-HPV was: HPV59 $(n=32$, $7.6 \%)$ HPV31 $(n=31,7.4 \%)$, HPV56 $(n=29,6.9 \%)$, HPV58 $(n=28,6.7 \%)$, HPV18 $(n=22,5.3 \%)$, HPV35 $(n=18,4.3 \%), \operatorname{HPV} 45(n=18,4.3 \%), \operatorname{HPV} 68(n=17$, $4.1 \%)$, and HPV33 ( $n=12,2.9 \%)$. Participants with measured HPV genotypes and DMR methylation data were comparable to those of the entire cohort with respect to age, HPV infection, yearly income, marital status and cigarette smoking (all $p>0.05$ ).

The median age was 28.6 years (IQR 11.6) and did not differ significantly across the three CIN groups. Overall, $82.8 \%(n=347)$ of women had $\leq$ CIN1, $12.4 \%(n=52)$ had CIN2 and 4.8\% $(n=20)$ had CIN3+ (Table 1$)$. Highrisk HPV infection prevalence was $70.9 \%$ in women with $\leq$ CIN1, $84.6 \%$ in CIN2 and $95 \%$ in CIN3 $(p=.07)$. Women with CIN2+ were more likely to be parous than those with $\leq \mathrm{CIN} 1(p=.003)$, and to be current smokers $(p<0001)$. There were no significant differences by ethnicity or contraceptive use across the categories $(p>.05)$.

\section{Association between CpG methylation and CIN}

Median methylation levels in CIN3+ vs $\leq \mathrm{CIN} 2$ and CIN2+ vs $\leq$ CIN1 varied across DMRs (Additional file 2). The ROC curve analysis of DMR methylation identification of CIN2+ and CIN3+ are shown in Additional file 3. The ROC area under the curve (AUC) values for CIN2+ were low for all DMRs, ranging from 0.54 (95\%CI: 0.460.62 ) in IGF2AS to 0.50 (95\%CI: 0.42-0.57) in MESTITI/ $M E S T$. The ROC AUC values for CIN3+ were also low, ranging from $0.62(95 \% \mathrm{CI}: 0.51-0.73)$ in $M E G 3$ to 0.51 (95\%CI: 0.38-0.64) in PEG10.

The multivariable-adjusted odds ratios and 95\% CI for the associations between DMR methylation and CIN status, adjusted for age, HPV infection, race, smoking and parity are shown in Table 2. Comparing CIN2+ to $\leq$ CIN1 we found no statistically significant associations between DMR methylation and CIN2+. Comparing CIN3+ to $\leq \mathrm{CIN} 2$, we found no statistically significant associations with methylation changes. No significant

Table 1 Characteristics of 419 study participants by cervical intraepithelial neoplasia (CIN) status

\begin{tabular}{|c|c|c|c|c|}
\hline Characteristic & $\begin{array}{l}\leq \mathrm{CIN} 1 \\
(n=347) \\
\mathrm{n}(\%)\end{array}$ & $\begin{array}{l}\text { CIN2 } \\
(n=52) \\
\mathrm{n}(\%)\end{array}$ & $\begin{array}{l}\text { CIN3+ } \\
(n=20) \\
n(\%)\end{array}$ & $p$ value \\
\hline Age (median, IQR) & $28.5(11.5)$ & $27.6(11.6)$ & $31.5(15.1)$ & .23 \\
\hline HPV infection & & & & .07 \\
\hline None & $34(9.8)$ & $3(5.8)$ & $0(0.0)$ & \\
\hline High-risk & $246(70.9)$ & $44(84.6)$ & $19(95.0)$ & \\
\hline Low-risk & $67(19.3)$ & $5(9.6)$ & $1(5.0)$ & \\
\hline Ethnicity & & & & .59 \\
\hline Non-Hispanic Black & $145(41.8)$ & $18(34.6)$ & $5(25.0)$ & \\
\hline Non-Hispanic White & $166(47.8)$ & $27(51.9)$ & $13(65.0)$ & \\
\hline Hispanic & $16(4.6)$ & $4(7.7)$ & $1(5.0)$ & \\
\hline Other $^{a}$ & $20(5.8)$ & $3(5.8)$ & $1(5.0)$ & \\
\hline Current cigarette smoking ${ }^{\mathrm{b}}$ & & & & $<.0001$ \\
\hline Yes & $46(13.3)$ & $19(36.5)$ & $7(35.0)$ & \\
\hline No & $301(86.7)$ & $33(63.5)$ & $13(65.0)$ & \\
\hline Parity $^{b}$ & & & & .003 \\
\hline Nulliparous & $186(53.6)$ & $18(35.3)$ & $6(30.0)$ & \\
\hline Parous & $161(46.4)$ & $33(64.7)$ & $14(70.0)$ & \\
\hline Hormonal contraceptive use ${ }^{b}$ & & & & .26 \\
\hline Yes & $260(79.8)$ & $35(77.8)$ & $13(72.2)$ & \\
\hline No & $66(20.2)$ & $10(22.2)$ & $5(27.8)$ & \\
\hline
\end{tabular}


Table 2 Odds ratios (OR) and 95\% confidence intervals (CI) for associations between methylation levels of differentially methylated regions (DMR) regulating genomically imprinted genes and CIN

\begin{tabular}{|c|c|c|c|c|c|c|}
\hline \multirow[b]{2}{*}{ Regulatory DMR } & \multicolumn{2}{|l|}{$\begin{array}{l}\text { All, } N=419 \\
\operatorname{OR}^{\mathrm{a}}(95 \% \mathrm{Cl})\end{array}$} & \multicolumn{2}{|l|}{$\begin{array}{l}\text { Black, } N=168 \\
\text { OR }^{\mathrm{a}}(95 \% \mathrm{Cl})\end{array}$} & \multicolumn{2}{|l|}{$\begin{array}{l}\text { White, } N=206 \\
\text { OR }^{\mathrm{a}}(95 \% \mathrm{Cl})\end{array}$} \\
\hline & CIN2+ vs. $\leq \mathrm{CIN} 1$ & CIN3+ vs. $\leq$ CIN2 & CIN2+ vs. $\leq$ CIN1 & CIN3+ vs. $\leq \mathrm{CIN} 2$ & CIN2+ vs. $\leq$ CIN1 & CIN3+ vs. $\leq$ CIN2 \\
\hline$\overline{P E G 3}$ & $1.16(0.87-1.56)$ & $0.79(0.48-1.31)$ & $1.31(0.76-2.25)$ & $1.71(0.71-4.10)$ & $1.13(0.73-1.75)$ & $0.70(0.37-1.32)$ \\
\hline PLAGL1/HYMAI & $1.01(0.73-1.38)$ & $0.92(0.51-1.66)$ & $0.82(0.44-1.52)$ & $1.15(0.51-2.58)$ & $0.98(0.63-1.52)$ & $0.75(0.35-1.61)$ \\
\hline KV DMR & $1.21(0.80-1.83)$ & $0.91(0.43-1.89)$ & $1.22(0.68-2.17)$ & $1.47(0.67-3.21)$ & $1.58(0.74-3.35)$ & $0.88(0.32-2.26)$ \\
\hline IGF2/H19 & $1.15(0.72-1.82)$ & $0.76(0.41-1.42)$ & $0.43(0.17-1.06)$ & $0.43(0.14-1.31)$ & $1.61(0.85-3.06)$ & $0.93(0.44-2.00)$ \\
\hline IGF2AS & $1.13(0.93-1.38)$ & $1.16(0.82-1.65)$ & $1.20(0.85-1.71)$ & $1.22(0.73-2.03)$ & $1.12(0.85-1.48)$ & $1.08(0.72-1.61)$ \\
\hline MESTITI/MEST & $1.08(0.74-1.58)$ & $0.90(0.48-1.70)$ & $1.14(0.59-2.20)$ & $0.36(0.13-1.98)$ & $0.88(0.52-1.47)$ & $1.11(0.51-2.42)$ \\
\hline PEG10 & $1.15(0.73-1.81)$ & $1.04(0.48-2.23)$ & $1.25(0.50-3.13)$ & $1.73(0.45-6.74)$ & $1.00(0.54-1.85)$ & $0.82(0.32-2.08)$ \\
\hline MEG3 & $1.01(0.79-1.28)$ & $1.38(0.95-1.99)$ & $0.95(0.63-1.44)$ & $1.06(0.60-1.88)$ & $1.13(0.80-1.62)$ & $1.58(0.97-2.58)$ \\
\hline
\end{tabular}

Abbreviations: CIN Cervical intraepithelial neoplasia, DMR Differentially methylated region, OR Odds ratio

${ }^{a}$ OR adjusted for age, high risk HPV, race, smoking and parity

In stratified analyses, ORs for other races (Hispanic/Asian/Pacific Islanders/Native Americans) not shown $(n=45)$

associations were found when stratified by race. In additional analysis, we restricted the outcome to HR-HPV positive, p16 positive CIN2+ cases. In our study $47 \%$ of CIN2+ cases were stained for p16, and of these, 30/34 (88\%) were positive. Comparing HR-HPV positive, p16+, CIN2+ cases to p16 negative/missing, $\leq$ CIN2 cases, we found a statistically significant association with PEG3 methylation (OR: 1.56 95\% CI: 1.03-2.36) (Additional file 4). No other significant associations were found.

\section{Association between HPV infection and CpG methylation} High-risk HPV infections were significantly associated with altered methylation levels at PLAGL1/HYMAI DMR $(\beta=-2.25, \mathrm{SE}=0.92, p<.01)$ (Table 3$)$. HR-HPV infections were also associated with IGF2/H19 $(\beta=2.49$, $\mathrm{SE}=1.37, p=.07)$, MESTIT1 $/ M E S T(\beta=1.46, \mathrm{SE}=0.83$, $p=.08)$ and PEG10 $(\beta=0.84, \mathrm{SE}=0.5, p=.09)$ DMRs but these were not statistically significant. Among black women, the association between HR-HPV and methylation in DMRs was strongest for PLAGL1/HYMAI DMR $(\beta=-2.68, \mathrm{SE}=1.34, p=.04)$ and $K v \operatorname{DMR}(\beta=1.80$, $\mathrm{SE}=0.81, p=.03)$. Among white women, the strongest association between HR-HPV infection and methylation was at MEG3 DMR $(\beta=3.82, \mathrm{SE}=1.04, p<.01)$.

\section{Discussion}

In this multiethnic cohort of women, we found a statistically significant association between altered methylation at PEG3 DMR and CIN2+ in HR-HPV positive cases. No other statistically significant associations were found.

Studies have shown that CIN2+ and CIN3+ have higher levels of DNA methylation than CIN1 [10, 14]. A study summarizing the performance of DNA methylation of human genes and HPV virus in detecting CIN2+ and CIN3+ found that the DNA markers had high

Table 3 Regression coefficients and standard errors (SE) for the association between high risk and low risk HPV infections and DMR methylation

\begin{tabular}{|c|c|c|c|c|c|c|}
\hline \multirow[t]{2}{*}{ Regulatory DMR } & \multicolumn{2}{|l|}{$\begin{array}{l}\text { All, } N=419 \\
\text { Coefficients (SE) }\end{array}$} & \multicolumn{2}{|l|}{$\begin{array}{l}\text { Blacks, } N=168 \\
\text { Coefficients (SE) }\end{array}$} & \multicolumn{2}{|l|}{$\begin{array}{l}\text { Whites, } N=206 \\
\text { Coefficients (SE) }\end{array}$} \\
\hline & HR-HPV & LR-HPV & HR-HPV & LR-HPV & HR-HPV & LR HPV \\
\hline PEG3 & $-0.30(0.77), p=.69$ & $1.44(0.90), p=.11$ & $0.04(1.21), p=.97$ & $2.51(1.47), p=.09)$ & No convergence & $-1.21(1.29), p=.35$ \\
\hline PLAGL1/HYMAI & $-2.85(0.92), p<.01$ & $-1.55(1.08), p=.15$ & $-2.68(1.34), p=.04$ & $-1.50(1.68), p=.37$ & $-1.95(1.39), p=.16$ & $-0.58(1.60), p=.71$ \\
\hline KV DMR & $0.37(0.50), p=.47$ & $0.33(0.59), p=.58$ & $1.80(0.81), p=.03$ & $2.51(0.96), p<.01$ & $-0.87(0.74), p=.24$ & $-1.39(0.86), p=.11$ \\
\hline IGF2/H19 & $2.49(1.37), p=.07$ & $2.80(1.58), p=.08$ & $3.02(2.27), p=.18$ & $3.11(2.73), p=.25$ & $2.84(1.92), p=.14$ & $2.84(2.18), p=.19$ \\
\hline IGF2AS & $1.91(1.35), p=.16$ & $1.91(1.56), p=.22$ & $1.27(2.13), p=.55$ & $0.82(2.54), p=.75$ & $1.86(1.98), p=.34$ & $2.49(2.22), p=.26$ \\
\hline MESTIT1/MEST & $1.46(0.83), p=.08$ & $0.95(0.96), p=.33$ & $-0.94(1.37), p=.49$ & $-1.55(1.60), p=.33$ & $2.23(1.20), p=.06$ & $1.68(1.39), p=.23$ \\
\hline PEG10 & $0.84(0.50), p=.09$ & $2.00(0.59), p<.01$ & $0.83(0.78), p=.28$ & $1.35(0.94), p=.14$ & $0.72(0.75), p=.33$ & $2.05(0.85), p=.01$ \\
\hline MEG3 & $-0.17(1.17), p=.88$ & $-2.08(1.41) p=.14$ & $-0.17(1.17), p=.89$ & $-2.08(1.41), p=.14$ & $3.82(1.04), p<.01$ & $3.25(1.17), p<.01$ \\
\hline
\end{tabular}

Abbreviations: DMR Differentially methylated regions. HR-HPV High-risk human papillomavirus, LR-HPV Low-risk human papillomavirus, SE Standard error Mixed models allowed for adjustment of individual CpGs at each DMR

No HPV infection served as the referent category 
specificity to detect CIN lesions that were likely to progress [10].

We previously reported on the link between higher methylation of the PEG3 DMR and ICC in Tanzanian women [15]. Our results further support the hypothesis that aberrant methylation of PEG3 DMR may be an important factor in the CIN progression in HR-HPV positive women, with $10 \%$ increment in methylation at PEG3 DMR associated with 1.56 times the odds of CIN2+. Evidence suggests that $P E G 3$ plays an important biological role in P53/c-myc mediated apoptosis, implicating PEG3 functions as a tumor suppressor in carcinogenesis [29, 30].

The odds ratio for the association between methylation in IGF2/H19 DMR and HR-HPV positive CIN2+ was 1.62 (95\%CI: $0.82-3.20)$. This was not statistically significant. In the Tanzania study, we reported a significant association between methylation in IGF2/H19 imprinted domain and risk of CIN (OR: 1.51 95\%CI: 1.00-2.50) and ICC (OR: 2.00 95\%CI:1.14-3.44) [16]. The difference in findings may be explained in part by the difference in comparison groups and outcomes in the two studies. The Tanzania study distinguished between the presence of CIN vs. no CIN and ICC vs no $\mathrm{CIN}$, whereas, in the current study, the primary comparison was CIN2+ vs CIN1. Furthermore, the most advanced outcome in our study was CIN3 compared to ICC in the previous study. Mechanistically, aberrant methylation in some CpGs in the IGF2/H19 imprinted domain may influence loss of imprinting and the exclusive use of IGF2 promoter 1, inducing IGF2 overexpression as previously shown [31]. The Tanzania study also showed a statistically significant association between HR-HPV infection and IGF2 DMR methylation $(\beta=$ $-8.55, p<.0001)$, supporting the hypothesis that aberrant DNA methylation may mediate the association between HR-HPV infection and the risk of high grade CIN and ICC. This association was not statistically significant in our study $(\beta=2.49, p=.07)$. Earlier analyses in this cohort, reported a negative association between aberrant DNA methylation of IGF2AS DMR and the regression of low-grade cervical lesions ( $\mathrm{HR}=0.41 \quad 95 \% \mathrm{CI}=0.23$ 0.76) [17]. We found no statistically significant association with high-grade lesions in our analyses.

A limitation of this study is its cross-sectional nature which limits the ability to infer methylation in DMRs as important factors in disease progression. However, identifying methylation markers associated with $\mathrm{CIN} 2+$ is an important step to allow for longitudinal evaluation of these markers in CIN progression. Secondly, we had few cases of CIN3+ and no cases of ICC in this study, which could have affected our effect estimates. In prior studies, we reported statistically significant associations between DMR methylation and ICC, and corresponding significant associations between DMR methylation and HR-HPV infections $[15,16]$. Although we had CIN2+ cases ( $n=52$ CIN2 and 20 CIN3 cases) that allowed us to analyze by grade-specific CIN, research shows that approximately $40 \%$ of undiagnosed CIN2 cases will regress over time [32, 33]. Furthermore, the grading of cervical lesions, particularly CIN1 and CIN2 could be affected by discordant grading among pathologists [34]. P16 staining has shown better concordance in diagnosis [35]. However, in our study, $47 \%$ of the CIN2+ cases were stained. Thus, we cannot rule out some misclassification in grading of cervical lesions. Lastly, we were limited in statistical power to examine DMR methylation in relation to grade and ethnic-specific $\mathrm{CIN} 2+$, after accounting for the effect of HPV infection. Cytosine methylation at imprinted genes is normally a stable modification in human tissue samples, and therefore DMR methylation status could potentially be used as a marker to identify high-grade lesions that are likely to progress to ICC.

In summary, aberrant DNA methylation at the PEG3 DMR may be associated with CIN2+ in HR-HPV positive cases. A study with sufficient cases of CIN3+ could confirm whether DNA methylation at this DMR represents susceptibility loci that could be exploited to identify CIN2+ cases that are likely to progress.

\section{Conclusions}

DNA methylation analysis is a promising risk stratification strategy to distinguish HR-HPV positive women with clinically relevant cervical lesions from those with non-progressive infections. Our findings suggest that PEG3 methylation may be associated with advanced cervical CIN lesions. Further investigations are warranted to determine its efficacy as a biomarker for cervical cancer screening.

\section{Abbreviations}

AUC: Area under the curve; Cl: Confidence intervals; CIN: Cervical intraepithelial neoplasia; CINCS: Cervical Intraepithelial Neoplasia Cohort Study; DMR: Differentially methylated region; HPV: Human papillomavirus; HR-HPV: High-risk human papillomavirus; ICC: Invasive cervical cancer; IQR: Interquartile range; LSIL: Low grade squamous intraepithelial lesion; LRHPV: Low-risk human papillomavirus; OR: Odds ratio; ROC: Receiver operating characteristic; SE: Standard error

\section{Supplementary Information}

The online version contains supplementary material available at https://doi. org/10.1186/s13027-021-00382-3.

Additional file 1: Supplementary Table 1. Primers used for Sequenom analysis.

Additional file 2: Supplementary Table 2. Median DMR methylation levels expressed as percentages by cervical intraepithelial neoplasia (CIN) status. 
Additional file 3: Supplementary Table 3. Crude odds ratios and receiver operator characteristic (ROC) curves for the association between the different DMRs and CIN status.

Additional file 4: Supplementary Table 4. Odds ratios (OR) and 95\% confidence intervals (CI) for association between DMR methylation markers and CIN2+ cases with HR-HPV infection and p16 positive.

\section{Acknowledgements}

Not applicable.

\section{Authors' contributions}

The authors contributed substantially to the study conception, design or acquisition of the data (D.S., F.V., R.B., M.G., S.K.M., C.H.); study conduct, data collection, or laboratory analysis (Z.H., D.S., F.V., R.B., M.G., K.S.H.Y., A.F., F.O., C.H.); data analysis and interpretation (C.B, A.C.V., J.S.S., S.K.M., C.H.); manuscript preparation (C.B., A.C.V., J.S.S., C.H.); critical review of manuscript (C.B., A.C.V., J.S.S., D.J., Z.H., R.B., M.G., K.S.H.Y., A.F., F.O., S.K.M., C.H.). All authors have reviewed and approved the final manuscript.

\section{Funding}

This study was supported in part by a grant from the National Cancer Institute (R01CA142983) to C.H. and S.K.M. and a supplement to A.C.V. The funder played no role in the design of the study, collection, analysis, and interpretation of data. C.H. was supported by a grant from National Institute on Minority Health and Health Disparities (R01MD011746). A.C.V. was supported by a Research Scholar Grant from the American Cancer Society (RSG-18-018-01-CPHPS). C.B. was supported by a National Cancer Institute Cancer Health Disparities training grant (T32-CA128582).

\section{Availability of data and materials}

The datasets analyzed during the current study available from the corresponding author on reasonable request.

\section{Declarations}

\section{Ethics approval and consent to participate}

This study was approved by Duke University School of Medicine Institutional Review Board (Pro\#00022943). Informed consent was obtained from each study participant prior to enrollment in the study.

\section{Consent for publication}

Not applicable.

\section{Competing interests}

J.S.S has received research grants and consultancies from BD Diagnostics and Hologic, and supply donations from Rovers and Arbor Vita over the past five years. Other co-authors have no potential conflicts of interest to declare.

\section{Author details}

${ }^{1}$ Department of Health Behavior, Gillings School of Global Public Health, University of North Carolina, Chapel Hill, NC, USA. ²Department of Surgery, Cedars-Sinai Medical Center, Los Angeles, CA, USA. ${ }^{3}$ Department of Epidemiology, Gillings School of Global Public Health and Lineberger Comprehensive Cancer Center, University of North Carolina, Chapel Hill, NC, USA. ${ }^{4}$ Department of Biological Sciences, Center for Human Health and the Environment, North Carolina State University, Raleigh, NC, USA. ${ }^{5}$ Department of Obstetrics and Gynecology, Division of Reproductive Sciences, Duke University School of Medicine, Durham, NC, USA. ${ }^{6}$ Department of Obstetrics and Gynecology, Virginia Tech Carilion School of Medicine, Roanoke, VA, USA. ${ }^{7}$ Department of Pathology, Duke University School of Medicine, Durham, NC, USA. ${ }^{8}$ Department of Family Medicine and Community Health, Duke University School of Medicine, Durham, NC, USA.

Received: 19 October 2020 Accepted: 2 June 2021

Published online: 13 June 2021

\section{References}

1. American College of Obstetricians and Gynecologists. Practice bulletin no. 168: cervical cancer screening and prevention. Obstet Gynecol. 2016;128(4): e111-e30.
2. Siegel RL, Miller KD, Jemal A. Cancer statistics, 2020. CA Cancer J Clin. 2020; 70(1):7-30.

3. Howlader N, Noone AM, Krapcho M, Miller D, Brest A, Yu M, Ruhl J, Tatalovich Z, Mariotto A, Lewis DR, Chen HS, Feuer EJ, Cronin KA (eds). SEER Cancer Statistics Review, 1975-2018. Bethesda: National Cancer Institute. 2021. Available at:https://seer.cancer.gov/csr/1975_2018. Accessed May 21 2021.

4. Curry SJ, Krist AH, Owens DK, Barry MJ, Caughey AB, Davidson KW, et al. Screening for cervical cancer: US preventive services task force recommendation statement. JAMA. 2018;320(7):674-86. https://doi.org/10.1 001/jama.2018.10897.

5. Ogilvie GS, van Niekerk D, Krajden M, Smith LW, Cook D, Gondara L, et al. Effect of screening with primary cervical HPV testing vs cytology testing on high-grade cervical intraepithelial neoplasia at 48 months: the HPV FOCAL randomized clinical trial. JAMA. 2018;320(1):43-52. https://doi.org/10.1001/ja ma.2018.7464

6. Koliopoulos G, Nyaga VN, Santesso N, Bryant A, Martin-Hirsch PP, Mustafa RA, et al. Cytology versus HPV testing for cervical cancer screening in the general population. Cochrane Database Syst Rev. 2017:8:CD008587.

7. Melnikow J, Henderson JT, Burda BU, Senger CA, Durbin S, Soulsby MA. Screening for cervical cancer with high-risk human papillomavirus testing: a systematic evidence review for the U.S. Prev Serv Task Force. 2018.

8. Wikström I, Lindell M, Sanner K, Wilander E. Self-sampling and HPV testing or ordinary pap-smear in women not regularly attending screening: a randomised study. Br J Cancer. 2011;105(3):337-9. https://doi.org/10.1038/ bjc.2011.236.

9. Lorincz AT. Cancer diagnostic classifiers based on quantitative DNA methylation. Expert Rev Mol Diagn. 2014;14(3):293-305. https://doi.org/10.1 586/14737159.2014.897610.

10. Kelly H, Benavente Y, Pavon MA, De Sanjose S, Mayaud P, Lorincz AT. Performance of DNA methylation assays for detection of high-grade cervical intraepithelial neoplasia (CIN2+): a systematic review and meta-analysis. Br J Cancer. 2019;121(11):954-65. https://doi.org/10.1038/s41416-019-0593-4.

11. Feinberg AP, Koldobskiy MA, Göndör A. Epigenetic modulators, modifiers and mediators in cancer aetiology and progression. Nat Rev Genet. 2016; 17(5):284-99. https://doi.org/10.1038/nrg.2016.13.

12. Mai M, Qian C, Yokomizo A, Tindall DJ, Bostwick D, Polychronakos C, et al. Loss of imprinting and allele switching of p73 in renal cell carcinoma. Oncogene. 1998;17(13):1739-41. https://doi.org/10.1038/sj.onc.1202099.

13. Kazanets A, Shorstova T, Hilmi K, Marques M, Witcher M. Epigenetic silencing of tumor suppressor genes: paradigms, puzzles, and potential. Biochim Biophys Acta. 2016;1865(2):275-88.

14. Kremer WW, Steenbergen R, Heideman D, Kenter GG, Meijer C. The use of host cell DNA methylation analysis in the detection and management of women with advanced cervical intraepithelial neoplasia: a review. BJOG. 2021;128(3):504-14. https://doi.org/10.1111/1471-0528.16395.

15. Nye MD, Hoyo C, Huang Z, Vidal AC, Wang F, Overcash F, et al. Associations between methylation of paternally expressed gene 3 (PEG3), cervical intraepithelial neoplasia and invasive cervical cancer. PLoS One. 2013;8(2): e56325. https://doi.org/10.1371/journal.pone.0056325.

16. Vidal AC, Henry NM, Murphy SK, Oneko O, Nye M, Bartlett JA, et al. PEG1/MEST and IGF2 DNA methylation in CIN and in cervical cancer. Clin Transl Oncol. 2014;16(3):266-72. https://doi.org/10.1007/s12094013-1067-4

17. Gomih A, Smith JS, North KE, Hudgens MG, Brewster WR, Huang Z, et al. DNA methylation of imprinted gene control regions in the regression of low-grade cervical lesions. Int J Cancer. 2018;143(3):552-60. https://doi.org/1 0.1002/ijc.31350.

18. Song JY, Lee JK, Lee NW, Jung HH, Kim SH, Lee KW. Microarray analysis of normal cervix, carcinoma in situ, and invasive cervical cancer: identification of candidate genes in pathogenesis of invasion in cervical cancer. Int J Gynecol Cancer. 2008;18(5):1051-9. https://doi.org/10.1111/j.1525-1438.2007. 01164.x.

19. Zhang J, Yao T, Lin Z, Gao Y. Aberrant methylation of MEG3 functions as a potential plasma-based biomarker for cervical cancer. Sci Rep. 2017;7(1): 6271. https://doi.org/10.1038/s41598-017-06502-7.

20. Rodriguez BA, Weng YI, Liu TM, Zuo T, Hsu PY, Lin CH, et al. Estrogenmediated epigenetic repression of the imprinted gene cyclin-dependent kinase inhibitor 1C in breast cancer cells. Carcinogenesis. 2011;32(6):812-21. https://doi.org/10.1093/carcin/bgr017. 
21. Nakano S, Murakami K, Meguro M, Soejima H, Higashimoto K, Urano T, et al. Expression profile of LIT1/KCNQ1OT1 and epigenetic status at the KvDMR1 in colorectal cancers. Cancer Sci. 2006;97(11):1147-54. https://doi.org/1 0.1111/j.1349-7006.2006.00305.x

22. Vidal AC, Smith JS, Valea F, Bentley R, Gradison M, Yarnall KS, et al. HPV genotypes and cervical intraepithelial neoplasia in a multiethnic cohort in the southeastern USA. Cancer Causes Control. 2014;25(8):1055-62. https:// doi.org/10.1007/s10552-014-0406-2.

23. Massad LS, Einstein MH, Huh WK, Katki HA, Kinney WK, Schiffman M, et al. 2012 updated consensus guidelines for the management of abnormal cervical cancer screening tests and cancer precursors. Obstet Gynecol. 2013: 121(4):829-46. https://doi.org/10.1097/AOG.0b013e3182883a34.

24. Gravitt PE, Peyton CL, Alessi TQ, Wheeler CM, Coutlee F, Hildesheim A, et al. Improved amplification of genital human papillomaviruses. J Clin Microbiol. 2000;38(1):357-61. https://doi.org/10.1128/JCM.38.1.357-361.2000.

25. Gravitt PE, Peyton CL, Apple RJ, Wheeler CM. Genotyping of 27 human papillomavirus types by using $L 1$ consensus $P C R$ products by a singlehybridization, reverse line blot detection method. J Clin Microbiol. 1998; 36(10):3020-7. https://doi.org/10.1128/JCM.36.10.3020-3027.1998.

26. Bosch FX, Manos MM, Munoz N, Sherman M, Jansen AM, Peto J, et al. Prevalence of human papillomavirus in cervical cancer: a worldwide perspective. International biological study on cervical cancer (IBSCC) study group. J Natl Cancer Inst. 1995;87(11):796-802. https://doi.org/10.1093/jnci/ 87.11.796.

27. Cogliano V, Baan R, Straif K, Grosse Y, Secretan B, El Ghissassi F. Carcinogenicity of human papillomaviruses. Lancet Oncol. 2005;6(4):204. https://doi.org/10.1016/S1470-2045(05)70086-3.

28. Apgar BS, Zoschnick L, Wright TC Jr. The 2001 Bethesda system terminology. Am Fam Physician. 2003;68(10):1992-8.

29. Johnson MD, Wu X, Aithmitti N, Morrison RS. Peg3/Pw1 is a mediator between p53 and Bax in DNA damage-induced neuronal death. J Bio Chem. 2002;277(25):23000-7. https://doi.org/10.1074/jbc.M201907200.

30. Deng Y, Wu X. Peg3/Pw1 promotes p53-mediated apoptosis by inducing Bax translocation from cytosol to mitochondria. Proc Natl Acad Sci U S A. 2000:97(22):12050-5. https://doi.org/10.1073/pnas.97.22.12050

31. Mathur SP, Mathur RS, Gray EA, Lane D, Underwood PG, Kohler M, et al. Serum vascular endothelial growth factor C (VEGF-C) as a specific biomarker for advanced cervical cancer: relationship to insulin-like growth factor II (IGF-II), IGF binding protein 3 (IGF-BP3) and VEGF-A [corrected]. Gynecol Oncol. 2005;98(3):467-83. https://doi.org/10.1016/j.ygyno.2005.05.003.

32. Castle PE, Schiffman M, Wheeler CM, Solomon D. Evidence for frequent regression of cervical intraepithelial neoplasia-grade 2. Obstet Gynecol. 2009;113(1):18-25. https://doi.org/10.1097/AOG.0b013e31818f5008.

33. Skorstengaard M, Lynge E, Suhr J, Napolitano G. Conservative management of women with cervical intraepithelial neoplasia grade 2 in Denmark: a cohort study. BJOG. 2020;127(6):729-736. https://doi.org/10.1111/1471-0528.1 6081.

34. Katki HA, Schiffman M, Castle PE, Fetterman B, Poitras NE, Lorey T, et al. Benchmarking CIN 3+ risk as the basis for incorporating HPV and pap cotesting into cervical screening and management guidelines. J Low Genit Tract Dis. 2013;17(5 Suppl 1):S28-35. https://doi.org/10.1097/LGT.0b013e31 8285423c.

35. Dijkstra MG, Heideman DA, de Roy SC, Rozendaal L, Berkhof J, van Krimpen $\mathrm{K}$, et al. p16(INK4a) immunostaining as an alternative to histology review for reliable grading of cervical intraepithelial lesions. J Clin Pathol. 2010;63(11): 972-7. https://doi.org/10.1136/jcp.2010.078634.

\section{Publisher's Note}

Springer Nature remains neutral with regard to jurisdictional claims in published maps and institutional affiliations.

Ready to submit your research? Choose BMC and benefit from:

- fast, convenient online submission

- thorough peer review by experienced researchers in your field

- rapid publication on acceptance

- support for research data, including large and complex data types

- gold Open Access which fosters wider collaboration and increased citations

- maximum visibility for your research: over $100 \mathrm{M}$ website views per year

At $\mathrm{BMC}$, research is always in progress.

Learn more biomedcentral.com/submissions 